\title{
Expansion of a multi-pronged safe sleep quality improvement initiative to three children's hospital campuses
}

Traci Leong ${ }^{1 \dagger}$, Kerryn Roome ${ }^{2 \dagger}$, Terri Miller ${ }^{3+}$, Olivia Gorbatkin ${ }^{2+}$, Lori Singleton ${ }^{4,5+}$, Maneesha Agarwal ${ }^{2,4+}$ and Sarah Gard Lazarus ${ }^{4,6^{*}+}$

From 24th Annual Injury Free Coalition for Kids Conference: Forging New Frontiers: Motor Vehicle Safety for All Ages Fort Lauderdale, FL, USA. 06-08 December 2019

\begin{abstract}
Background: The American Academy of Pediatrics (AAP) recommends infants should be Alone, on their Back, and in a clear Crib to combat relatively stagnant rates of sudden unexpected infant death (SUID). These are referred to as the ABCs of safe sleep. Studies have shown these recommendations are not consistently followed in the hospital setting, but further investigation would determine how to improve the rate of adherence. The objective of this study was to evaluate the impact of an expanded safe sleep initiative at three Georgia free-standing children's hospital campuses before and after a multipronged safe sleep initiative.

Methods: A quality improvement program with a pre/post analysis was performed using a convenience method of sampling. Infants $<12$ months old in three inpatient pediatric campuses were analyzed pre- and post- interventions. The intervention included: 1) nursing education, 2) identification of nurse "safe sleep" champions, 3) crib cards, 4) crib audits, and 5) weekly reporting of data showing nursing unit $A B C$ compliance via tracking boards. The goal was $A B C$ compliance of $\geq 25 \%$ for the post-intervention period. A standardized crib audit tool evaluated sleep position/location, sleep environment, and ABC compliance (both safe position/location and environment). Chi square analysis, Fisher's exact test, and logistic regression were used to compare safe sleep behaviors before and after the interventions.

Results: There were 204 cribs included pre-intervention and 274 cribs post-intervention. Overall, there was not a significant change in sleep position/location $(78.4$ to $76.6 \%, p=0.64)$. There was a significant increase in the percent of infants sleeping in a safe sleep environment following the intervention (5.9 to $39.8 \%, p<0.01$ ). Overall ABC compliance, including both sleep position/location and environment, improved from $4.4 \%$ pre-intervention to $32.5 \%$ postintervention $(p<0.01)$. There was no significant variability between the hospitals $(p=0.71, p=1.00)$.

Conclusions: The AAP's safe sleep recommendations are currently not upheld in children's hospitals, but safer sleep was achieved across three children's campuses in this study. Significant improvements were made in sleep environment and overall safe sleep compliance with this multi-pronged initiative.
\end{abstract}

Keywords: Infant mortality, Sleep safety, Sudden unexplained infant death, Quality improvement

\footnotetext{
* Correspondence: drsarahgard@gmail.com

†Traci Leong, Kerryn Roome, Terri Miller, Olivia Gorbatkin, Lori Singleton,

Maneesha Agarwal and Sarah Gard Lazarus contributed equally to this work.

${ }^{4}$ Children's Healthcare of Atlanta, Atlanta 30322, USA

${ }^{6}$ Pediatric Emergency Medicine Associates, Atlanta 30342, USA

Full list of author information is available at the end of the article
}

(c) The Author(s). 2020 Open Access This article is licensed under a Creative Commons Attribution 4.0 International License, which permits use, sharing, adaptation, distribution and reproduction in any medium or format, as long as you give appropriate credit to the original author(s) and the source, provide a link to the Creative Commons licence, and indicate if changes were made. The images or other third party material in this article are included in the article's Creative Commons licence, unless indicated otherwise in a credit line to the material. If material is not included in the article's Creative Commons licence and your intended use is not permitted by statutory regulation or exceeds the permitted use, you will need to obtain permission directly from the copyright holder. To view a copy of this licence, visit http://creativecommons.org/licenses/by/4.0/ The Creative Commons Public Domain Dedication waiver (http://creativecommons.org/publicdomain/zero/1.0/) applies to the data made available in this article, unless otherwise stated in a credit line to the data. 


\section{Background}

The Centers for Disease Control and Prevention (CDC) defines sudden unexpected infant death (SUID) as "the sudden death of an infant under 1 year of age that cannot be explained after thorough investigation." (Patton et al. 2015; Centers for Disease Control and Prevention 2019) SUID is routinely classified as: 1) sudden infant death syndrome (SIDS), 2) accidental suffocation and strangulation in bed (ASSB), or 3) death from unknown causes. Each year, approximately 4000 U.S. babies die from SUID. (Georgia Department of Public Health 2016) SUID is associated with unsafe sleep practices. (Erck Lambert et al. 2018; Moon 2016) Between 1990 and 1999, the SUID rate drastically declined following numerous safe sleep campaigns, of which the "Back to Sleep" campaign in 1994 was the most well-known. In 2012, the National Institute of Health (NIH) expanded their focus to include environmental recommendations (such as sleep location and environment) and renamed it the "Safe to Sleep" campaign. (Moon 2016; National Institute of Child Health and Human Development/National Institutes of Health Safe to Sleep Campaign 2019) Since 1997, SIDS deaths have become less common; however, infant death due to unknown causes and ASSB rates are stagnant. (Centers for Disease Control and Prevention 2019; Moon 2016; Shapiro-Mendoza 2017)

To reduce sleep deaths, the American Academy of Pediatrics' (AAP) ABCs of sleep are paramount: place infants Alone, on their Back and in a clear Crib with only a firm mattress and a tight fitting sheet. (Georgia Department of Public Health 2016) It has been shown caregivers are more likely to adopt a particular behavior if they are exposed to it in a trusted setting, such as a hospital. (Heitmann et al. 2017) However, hospitalized infants are often found in unsafe sleep environments. (Mason et al. 2013; Leong et al. 2019) Multiple studies have utilized crib audits to collect information such as patient age, sleep position/location and environment, followed by a quality improvement (QI) program in children's hospitals and birthing centers; they found that safe sleep practices could be greatly improved. (Mason et al. 2013; Leong et al. 2019; Macklin et al. 2019; Shadman et al. 2016; Macklin et al. 2016; Moon 2011)

In response to Georgia's high infant mortality rate, the Georgia Safe to Sleep campaign was initiated in 2016 to model and educate both parents and caregivers on safe sleep practices in mother-baby units at birthing hospitals. (Georgia Department of Public Health 2016) In 2017, we performed a study at a freestanding tertiary care children's hospital to study the effectiveness of a quality initiative aimed at safe sleep compliance before and after QI interventions. (Leong et al. 2019) We hypothesized baseline compliance with safe sleep recommendations on the general pediatric inpatient units in all three hospitals would be poor, but that there would be significant improvement with a multipronged quality initiative. The objectives of this study mirrored our prior study, the difference being a larger sample size, a greater variety in patient population, and expansion to multiple campuses.

The objectives were:

1) To assess baseline infant sleep behaviors at three children's hospital campuses

2) To evaluate the effectiveness of a multi-pronged quality improvement (QI) initiative that included nursing education, identification of nurse safe sleep champions, crib audits, crib cards with safe sleep checklists, and tracking boards in improving adherence to the $\mathrm{ABCs}$ of safe sleep at three children's hospital campuses

3) To demonstrate a successful safe sleep initiative can be expanded throughout a hospital system

\section{Methods \\ Study design}

This study is a pre-intervention and post-intervention study designed to determine the efficacy of a QI program focused on improving safe sleep practices in the inpatient setting of 3 children's hospitals. This study's methodology is based on a previous study by Leong et al., with the addition of two hospital campuses. (Leong et al. 2019) We evaluated infant safe sleep practices on inpatient general pediatric floors of two tertiary care hospitals and one community hospital, for a total of five inpatient floors using a validated crib audit tool. (Leong et al. 2019) The interventions of the study included: 1) nursing education, 2) identification of nurse "safe sleep" champions, 3) crib cards, 4) crib audits, and 5) weekly reporting of data showing $\mathrm{ABC}$ compliance via tracking boards. This study was found to be exempt by the hospital's institutional review board (IRB).

The general pediatric inpatient units of these hospitals were chosen due to a higher likelihood that they would represent the general pediatric population, in comparison to subspecialty units (e.g. mother/baby units). The crib audits were conducted on infants less than 12 months old in all three hospitals on the five general pediatric units. The pediatric units included in this QI program contained between 20 and 35 beds each, depending on the campus, with children ranging in age from 3 days to 21 years. Patients who were less than 12 months of age and asleep at the time of the crib audit were included in the study. Patients were excluded from the study if they were awake, intubated, had craniofacial anomalies, were requiring non-invasive positive pressure ventilation or high-flow nasal cannula, less than 32 weeks' gestation, had incomplete data in the crib audit, a current admission to an intensive care unit, or required isolette or temperature support. 


\section{Data collection}

Data were collected using the Georgia Department of Public Health's crib audit tool, which has been validated and utilized when evaluating the efficacy of the safe sleep programs at the birthing hospitals and in the previously performed study. (Leong et al. 2019) The crib audit tool was modified slightly to identify which campus and floor was audited. This tool is comprised of a checklist that keeps track of observations made in a patient's room. Our primary outcomes included sleep position/location, sleep environment, and overall ABC compliance. ABC compliance was defined as both safe sleep position/location and safe crib environment. A non-random, convenience sampling method was used.

If the child was awake, the investigators returned to the room after completing the crib audits for other infants on the unit and attempted the audit again. Data collection occurred over a time period of approximately 2 months for pre-intervention and 2 to 4 months for post-intervention (depending on campus location). Patients who had been audited on a previous day were not excluded, as each day was a new opportunity to assess sleep environment and position/location choices. Postintervention audits were collected on the same units as pre-intervention audits and using the same convenience sampling method.

The first assessment when doing the crib audits was determining if the infant was asleep or awake. If asleep, the crib audit was completed - assessing for position/location, sleep environment, and presence of caregiver. In order to be classified as having a safe sleep position/location, infants were required to be supine in a crib or held by an awake adult. Unsafe sleep position or location included prone positioning, sleeping in a caregiver's bed, being held by a sleeping adult, or sleeping in a device that was not a crib or bassinet (such as a swing).

The second assessment made during crib audits was the sleep environment. In order to be classified as having a safe sleep environment, the infant had to be alone in the sleeping environment. The presence in the sleeping environment of any soft or hard toys, extra blankets, medical equipment not in use, diapers, washcloths, clothing, or pillows were marked it as unsafe. Pacifiers, medical equipment in use, and/or a single swaddling blanket/sleep sack in use were allowed in the safe sleep environments. During this study, due to current hospital policy, head-of-bed elevation was still considered safe, if the infant met the position/location and environmental requirements.

\section{Intervention}

Once baseline safe sleep data were collected, the quality improvement initiative was implemented. Crib cards were made, and nurses were asked to place the cards on every crib for an infant under 12 months of age. The crib cards consisted of a checklist with the ABCs of safe sleep. Reminders were given during morning huddle. Safe sleep champions also encouraged nurses and staff to remove unsafe items. At the end of each week, tracking boards with baseline compliance on the unit to date were placed in each unit's nursing break room. The tracking boards included information on how they had progressed since the previous week, the proportion of infants who were compliant with the AAP's safe sleep recommendations, and the proportion of infants with correctly hung crib cards. These tracking boards were meant to act as encouragement as well as real-time feedback. Between $24 \mathrm{~h}$ and 7 days after the use of the crib cards and the tracking boards, post-intervention audits were collected. The sampling method and crib audit tool used in the pre-intervention time period were used again for post-intervention data collection. The postintervention goal for $\mathrm{ABC}$ compliance was 25\%. This goal was selected as a realistic target based on the preintervention $\mathrm{ABC}$ compliance at hospital 1.

\section{Statistical analysis}

Data were collected using REDCap $($, an online database. Descriptive frequencies were calculated (counts and percentages) to assess the safe sleep behaviors for both phases of the crib audits. Chi-square or Fisher's exact test (when appropriate) was used to assess safe sleep behaviors for both the pre-intervention and postintervention periods. Three pre- and post-intervention comparisons were conducted to evaluate: 1) the proportion of infants in safe sleep position/location, 2) the proportion of infants sleeping alone, and 3) the proportion of infants with overall ABC compliance (safe positioning and environment). Logistic regression was used for both crib environment and overall $\mathrm{ABC}$ compliance to estimate the effect of each hospital and time periods. Interactions between hospital and time period were tested. Rv3.6.0 (Vienna, Austria) was used for statistical analysis and conducted using $R$ Core Team (Core Team 2018); figures were produced using the package ggplot2. (Wickham 2009)

\section{Results \\ Crib audits}

A total of 648 infants were screened among the three children's hospital campuses. We excluded 170 infants. Thus, 478 infants were included for the final analysis, with 204 pre-intervention and 274 post-intervention (Fig. 1).

\section{Sleep position and location}

A safe sleep position/location is defined as the infant on his/her back/supine or sleeping in the arms of an awake caregiver. Most infants both pre- and post-intervention were in a safe sleep position/location, $78.4 \%$ (95\% CI 


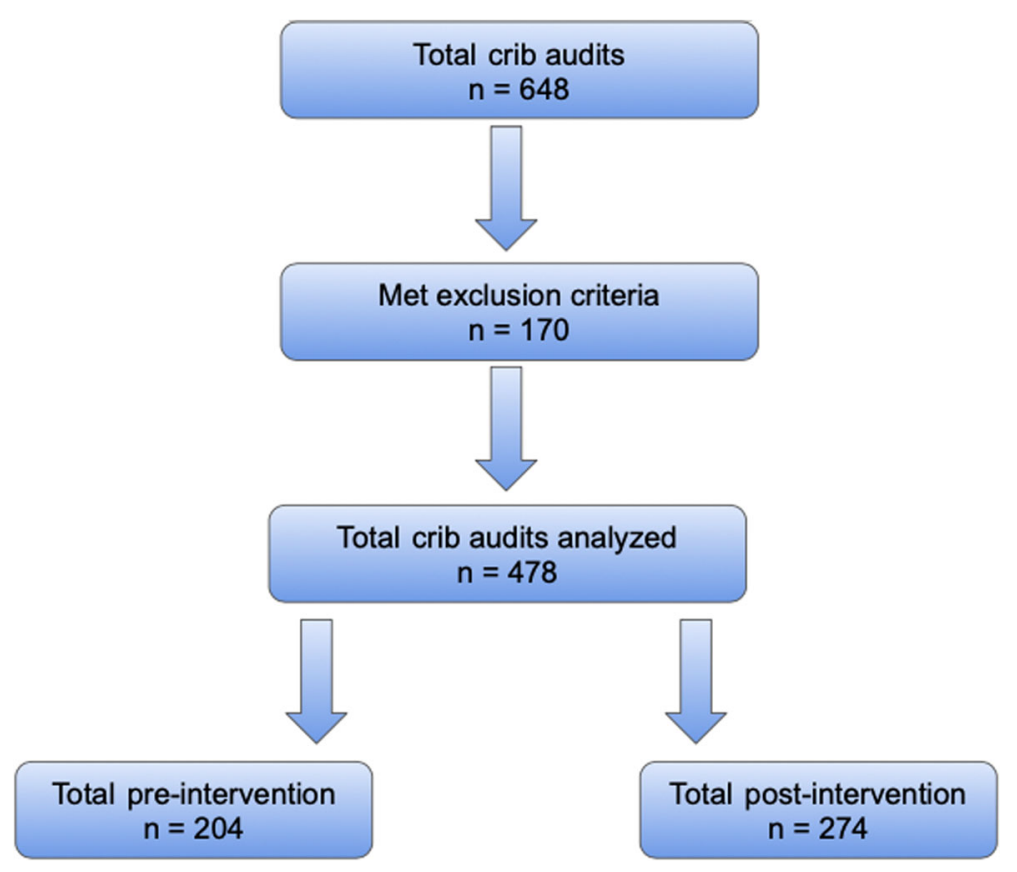

Fig. 1 Patient inclusion flow chart

$72.2-83.5 \%)$ and $76.6 \%$ (95\% CI 71.3-81.3\%) respectively (Table 1). There was no difference between pre- and postintervention periods $(p=0.64)$. When analyzing unsafe sleep positions/locations prior to intervention, $3.9 \%$ were prone, $4.4 \%$ were sleeping on/in a caregiver's bed, $3.4 \%$ were held by a sleeping adult, and $6.4 \%$ were side-sleeping. Following the intervention, $6.9 \%$ were prone, $7.3 \%$ were sleeping on/in a caregiver's bed, $4 \%$ were held by a sleeping adult, and $4.4 \%$ were side-sleeping (Table 1).When safe sleep position/location is compared by hospital, utilizing logistic regression, there is no significant difference between hospitals $(p=0.36)$.

\section{Sleep environment}

The crib environments, when combining the results of all three campuses, were significantly safer post intervention, 5.9\% (95\% CI 3.3-10.1\%) vs. 39.8\% (95\% CI 34.2$45.7 \%),(p<0.01)$ (Table 2). The specifics of the items found in the cribs are listed in Table 2 . There were statistically significant decreases in the following items found in the cribs post-intervention: clothing (22.1 to $6.6 \%, p<0.01$ ), diapers ( 21.6 to $4.7 \%, p<0.01)$, stuffed toy (23.5 to $10.6 \%, p<0.01$ ), burp cloths (16.7 to $2.6 \%, p<$ 0.01 ), extra blankets ( 74 to $38.7 \%, p<0.01$ ), suction bulbs (5.4 to $0.4 \%, p<0.01$ ), fluffy blankets (27 to $17.5 \%, p=$ 0.02 ), wipes (28.4 to $9.1 \%, p<0.01$ ), plastic toys (9.3 to $3.6 \%, p=0.01$ ), and medical equipment not in use (27.5 to $5.8 \%, p<0.01)$. There was no decrease in pillows and nonspecified loose items found in the cribs post-intervention (Table 2). When comparing hospitals, adjusting for period in the logistic regression model, there are significant differences by intervention, but not by hospital. The test for interaction was not significant, meaning, the effect of the specific hospital on the crib environment does not differ by pre-intervention and post-intervention.

\section{ABC compliance}

ABC compliance (defined as including both safe sleep position/location and safe crib environment) significantly improved collectively among the three hospitals from $4.4 \%(2.0-8.2 \%)$ in the pre-intervention period to $32.5 \%(27.2-38.2 \%)$ to the post-intervention period $(p<0.01)$ (Table 3). ABC compliance improved, even after adjusting for each hospital (OR 10.6, 95\% CI 5.4, 23.2). Of note, each campus met and exceeded the ABC compliance goal of $25 \%$ following the interventions. Figure 2 illustrates the changes found in all three categories: sleep position/location, sleep environment, and $\mathrm{ABC}$ compliance.

\section{Discussion}

In this expansion of a safe infant sleep QI program, overall compliance with $\mathrm{ABC}$ recommendations improved significantly post-intervention. ABC compliance encompasses both sleep position/location and sleep environment, and among these two categories, a significant improvement was found only in sleep environment. As expected, and shown previously, sleep position/location did not improve to the same degree as sleep environment. (Leong et al. 2019) This is most likely due to the 
Table 1 Evaluation of sleep position/location* pre- and post-intervention

\begin{tabular}{|c|c|c|c|}
\hline Variable & $n$ & Pre $(\boldsymbol{n}=204)$ & Post $(\boldsymbol{n}=274)$ \\
\hline Safe sleep position/location*, n (\%) & 478 & n (\%) & $\mathrm{n}(\%)^{* *}$ \\
\hline Not Safe & & $44(21.6)$ & $64(23.4)$ \\
\hline Safe & & $160(78.4)$ & $210(76.6)$ \\
\hline Sleep position/location, $\mathrm{n}(\%)$ & 478 & & \\
\hline Sleeping on back in crib & & $131(64.2)$ & $162(59.1)$ \\
\hline Sleeping on side in crib & & $13(6.4)$ & $12(4.4)$ \\
\hline Sleeping held by sleeping adult & & $7(3.4)$ & $11(4.0)$ \\
\hline Sleeping in another device & & $1(0.5)$ & 0 \\
\hline Sleeping on stomach in crib & & $8(3.9)$ & $19(6.9)$ \\
\hline Sleeping in Crib with Sleeping Adult & & $6(2.9)$ & $2(0.7)$ \\
\hline Sleeping on/in caregiver's bed & & $9(4.4)$ & $20(7.3)$ \\
\hline Sleeping held by an awake adult & & $29(14.2)$ & $48(17.5)$ \\
\hline
\end{tabular}

*Safe Sleep Position/location: In order to be classified as having a safe sleep position/location, infants were required to be supine in a crib or held by an awake adult

${ }^{* *} p=0.64$

Back to Sleep campaign being well known and in place for many years leading to greater knowledge of what a safe sleep position and location entails. (Shapiro-Mendoza 2017; Kuhlmann et al. 2016; Colson et al. 2017; de Luca and Hinde 2016) Unfortunately, despite overall good compliance, there was still a moderate prevalence of unsafe positioning. Around $20 \%$ of infants were still found to be in unsafe sleep positions. Prior to the intervention, the most common unsafe sleep position was side sleeping. Following the intervention, the most common unsafe sleep position was on/in a caregiver's bed.

Bed sharing has routinely been cited as a hazard to safe sleeping and the AAP recommends against it until 1 year of age, but especially in the newborn to six-month old age range. (Moon 2016) According to a 2015 study, $61 \%$ of caregivers report sharing a bed with their infant.

Table 2 Evaluation of crib environment ${ }^{a}$ pre- and post-intervention

\begin{tabular}{|c|c|c|c|}
\hline Variable & Pre $(\boldsymbol{n}=204)$ & Post $(\boldsymbol{n}=274)$ & $\boldsymbol{p}$-value \\
\hline Environment, n (\%) & n (\%) & n (\%) & $<0.01$ \\
\hline Not safe & $192(94.1)$ & $165(60.2)$ & \\
\hline Safe & $12(5.9)$ & $109(39.8)$ & \\
\hline Clothing, n (\%) & $45(22.1)$ & $18(6.6)$ & $<0.01$ \\
\hline Diapers, n (\%) & $44(21.6)$ & $13(4.7)$ & $<0.01$ \\
\hline Stuffed toy, n (\%) & $48(23.5)$ & $29(10.6)$ & $<0.01$ \\
\hline Burping cloths, n (\%) & $34(16.7)$ & $7(2.6)$ & $<0.01$ \\
\hline Pillow, n (\%) & $27(13.2)$ & $22(8.0)$ & 0.07 \\
\hline Extra blanket, n (\%) & $151(74.0)$ & $106(38.7)$ & $<0.01$ \\
\hline Suction, n (\%) & $11(5.4)$ & $1(0.4)$ & $<0.01$ \\
\hline Fluffy blanket, n (\%) & $55(27.0)$ & $48(17.5)$ & 0.02 \\
\hline Wipes, n (\%) & $58(28.4)$ & $25(9.1)$ & $<0.01$ \\
\hline Plastic toy, n (\%) & $19(9.3)$ & $10(3.6)$ & 0.01 \\
\hline Bottle, n (\%) & $7(3.4)$ & $10(3.6)$ & 1.0 \\
\hline Med equipment NOT in use, $\mathrm{n}(\%)$ & $56(27.5)$ & $16(5.8)$ & $<0.01$ \\
\hline Electronics, n (\%) & $1(0.5)$ & $4(1.5)$ & 0.40 \\
\hline Bumpers, n (\%) & $0(0.0)$ & $2(0.7)$ & 0.51 \\
\hline Books, n (\%) & $1(0.5)$ & $0(0.0)$ & 0.43 \\
\hline Other loose items, n (\%) & $2(1.0)$ & $0(0.0)$ & 0.18 \\
\hline
\end{tabular}

${ }^{a}$ Safe sleep environment defined as a clear crib except for pacifier and single swaddled blanket 
Table 3 Evaluation of ABC compliance pre- and postintervention

\begin{tabular}{lllr}
\hline Variable & Pre $(\boldsymbol{n}=204)$ & Post $(\boldsymbol{n}=274)$ & $\boldsymbol{p}$-value \\
\hline ABC Compliance $^{\mathrm{a}}, \boldsymbol{n}(\%)$ & $\mathrm{n},(\%)$ & $\mathrm{n},(\%)$ & $<0.01$ \\
No & $195(95.6)$ & $185(67.5)$ & \\
Yes & $9(4.4)$ & $89(32.5)$ & \\
\hline
\end{tabular}

${ }^{\mathrm{a} A B C}$ Compliance: compliant with both safe sleep position/location and environment

(Centers for Disease Control and Prevention 2018) While several studies have linked it to an increased risk of SUID, it is still common practice. We hypothesize this may be seen especially in the hospital setting when caregiver's feel the need to comfort their infant during a stressful time. In this study, the hospital policies allow for caregivers to sign a waiver allowing co-sleeping. In addition, this policy allows for replacing a crib with an adult-sized hospital bed, so the caregiver has a comfortable place to sleep with the infant. This is not standard hospital practice, and in the future, we hope to remove the co-sleeping waiver in order to discourage straying from the ABCs of safe sleep. It is clear additional education for caregivers on safe sleep is beneficial; however, it is unrealistic to expect caregivers to follow recommendations if healthcare professionals aren't following them. Thus, it is important to understand the level of adherence to safe sleep recommendations in hospitals with the goal of improving safe sleep practices.

Following the implementation of the program, sleep environment was found to be significantly safer on the inpatient pediatric units with fewer items found in the cribs. Many of the items found in the cribs prior to the intervention were soft in nature - stuffed toys, diapers, burping cloths, pillows, fluffy blankets, extra blankets, and clothing.
Loose, soft objects can obstruct airways and in doing so, increase the risk of SIDS and ASSB. (Moon 2016; Kanetake et al. 2003; Kemp et al. 1998; Patel et al. 2001; Shapiro-Mendoza et al. 2015; Scheers et al. 1998) The initial low compliance with a safe sleep environment supports the claim that the Back to Sleep campaign succeeded in increasing awareness about sleep position but did not result in universal adoption of best practices. It is clear more education and positive examples are needed. (Shadman et al. 2016; Macklin et al. 2016; Kuhlmann et al. 2016) Past studies suggest placing storage bins next to or at the end of cribs may be useful in encouraging the removal of nonessential items from the infant's crib. (Kuhlmann et al. 2016; McMullen et al. 2016; Zachritz et al. 2016) Despite significant decreases in certain items in cribs, an increase in items such as bottles, electronics, and bumpers were noted. This is likely due to the small sample size that reported these items. A study with a larger sample size would be needed to further analyze these items. To improve safe sleep compliance in the future, the development and implementation of a computer-based training (CBT) program, currently in process, may be useful.

\section{Limitations}

Data were collected from a convenience sample over a limited time period in a single state; however, 3 different hospitals were included. Because all interventions were performed at once, our statistics are reported as preand post-intervention results, as opposed to true PDSA cycles. Therefore, it is unclear which intervention had the greatest effect on compliance. If an infant was awake at the time of the audit, a second attempt was made to obtain the observational data. However, there was no

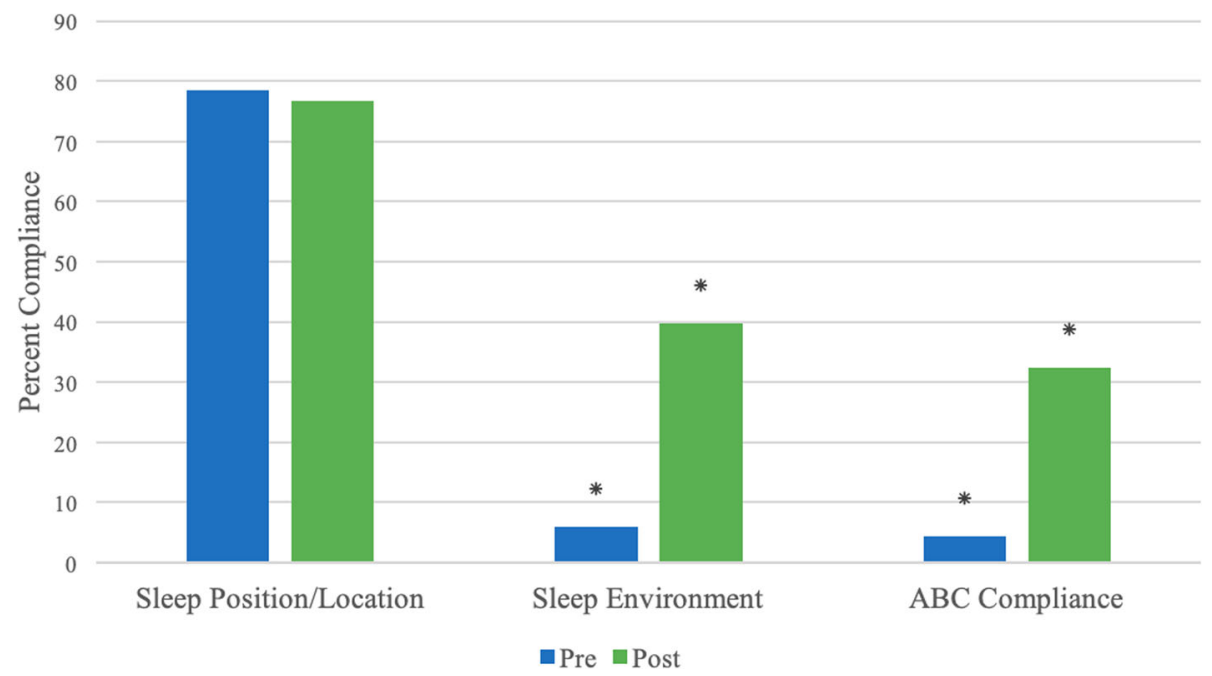

Fig. 2 Pre- and post-intervention comparisons of sleep position/location, environment, and overall $A B C$ compliance for all three campuses. ${ }^{*} p<$ 0.01. * represents a significant improvement 
required amount of time to wait between attempts, which may have led to a decrease in the potential sample size. Different reviewers performed audits and while the crib audit tool was made to be quite specific, different interpretations of certain items could have occurred. Crib card compliance was not consistently recorded in this study; therefore, it is difficult to determine the true impact of the crib cards. Another limitation of the study results is whether the interventions caused the significant increase in safe sleep or whether the Hawthorne Effect was heavily present. The changes in compliance found in this study may partly be the result of nurses and caregivers knowing they were being observed, and thus were more likely to follow safe sleep recommendations. We did not collect data on crib card compliance, so we cannot say that the compliance was high enough to combat the Hawthorne Effect.

\section{Conclusions}

A safe infant sleep QI program at three children's hospital campuses involving crib audits, nursing education, crib cards, and weekly tracking boards improved overall compliance with the ABCs of safe sleep. The program impacted sleep environment more than sleep position/ location, which we believe is due to the long history of supine sleep recommendations. It is still unclear what specific element of the intervention caused the significant improvement in safe sleep, but the multi-pronged approach created a safer sleep environment for hospitalized infants. Efforts to increase compliance with wellknown safe sleep recommendations in hospitals are needed, and long-term solutions such as storage bins and permanent safe sleep checklists may help.

\section{Abbreviations}

AAP: American Academy of Pediatrics; ASSB: Accidental Suffocation and Strangulation in Bed; CBT: Computer Based Training; CDC: Centers for Disease Control and Prevention; IRB: Institutional Review Board; NIH: National Institutes of Health; QI: Quality Improvement; SIDS: Sudden Infant Death Syndrome; SUID: Sudden Unexpected Infant Death; U.S.: United States

\section{Acknowledgments}

Thank you to Children's Healthcare of Atlanta and Pediatric Emergency Medicine Associates for their non-financial support of their project.

\section{About this supplement}

This article has been published as part of Injury Epidemiology Volume 7 Supplement 1, 2020: Proceedings from the 24th Annual Injury Free Coalition for Kids ${ }^{\oplus}$ Conference: Forging New Frontiers: Motor Vehicle Safety for All Ages. The full contents of the supplement are available online at https://injepijournal. biomedcentral.com/articles/supplements/volume-7-supplement-1.

\section{Authors' contributions}

TL performed data and statistical analysis and assisted with results section. KR cleaned data, wrote the first draft of the manuscript, and assisted with edits. MA assisted with edits and reviewed manuscript. TM assisted with conceptualization of design, reviewed the manuscript and made edits. OG performed hospital 2 audits and assisted with original analysis. LS assisted with edits and created run charts. SGL conceptualized design of study, assisted with data collection, collaborated and corresponded with authors, and performed edits. All authors read and approved the final manuscript.

\section{Funding}

There was no external funding for this research. Publication costs are funded by the Injury Free Coalition for Kids ${ }^{\mathrm{TM}}$.

\section{Availability of data and materials}

The datasets used and/or analyzed during the current study are available from the corresponding author on reasonable request.

\section{Ethics approval and consent to participate}

This study was deemed IRB exempt and need for ethics approval was waived.

\section{Consent for publication}

Not applicable.

\section{Competing interests}

The authors have no competing interests or financial relationships relevant to this article to disclose.

\section{Author details}

${ }^{1}$ Department of Biostatistics and Bioinformatics, Emory University Rollins School of Public Health, Atlanta 30322, USA. Emory University School of Medicine, Atlanta 30322, USA. ${ }^{3}$ Georgia Department of Public Health, Atlanta 30303, USA. ${ }^{4}$ Children's Healthcare of Atlanta, Atlanta 30322, USA.

${ }^{5}$ Morehouse School of Medicine, Atlanta 30310, USA. ${ }^{6}$ Pediatric Emergency Medicine Associates, Atlanta 30342, USA.

Published: 12 June 2020

\section{References}

Centers for Disease Control and Prevention. Vital Signs: Safe Sleep for Babies 2018. Available from: https://www.cdc.gov/vitalsigns/safesleep/index.html. Accessed on 15 Oct 2019

Centers for Disease Control and Prevention. Sudden Unexpected Infant Death and Sudden Infant Death Syndrome: Data and Statistics 2019. Available from: https://www.cdc.gov/sids/data.htm. Accessed on 12 Oct 2019.

Colson ER, Geller NL, Heeren T, Corwin MJ. Factors Associated With Choice of Infant Sleep Position. Pediatrics. 2017;140(3):1032-7.

Core Team R. R: a language and environment for statistical computing. Vienna: R Foundation for Statistical Computing; 2018. Available from: https://www.Rproject.org/. Accessed on 14 Oct 2019

de Luca F, Hinde A. Effectiveness of the 'Back-to-sleep' campaigns among healthcare professionals in the past 20 years: a systematic review. BMJ Open. 2016;6(9):e011435.

Erck Lambert AB, Parks SE, Shapiro-Mendoza CK. National and State Trends in Sudden Unexpected Infant Death: 1990-2015. Pediatrics. 2018;141(3). https:// doi.org/10.1542/peds.2017-3519.

Georgia Department of Public Health. SIDS Data and Risk Factors 2016 Updated 8 March 2016. Available from: https://dph.georgia.gov/sids-data-and-riskfactors. Accessed on 12 Oct 2019.

Heitmann R, Nilles EK, Jeans A, Moreland J, Clarke C, McDonald MF, et al. Improving safe sleep modeling in the hospital through policy implementation. Matern Child Health J. 2017;21(11):1995-2000.

Kanetake J, Aoki Y, Funayama M. Evaluation of rebreathing potential on bedding for infant use. Pediatr Int. 2003;45(3):284-9.

Kemp JS, Livne M, White DK, Arfken CL. Softness and potential to cause rebreathing: differences in bedding used by infants at high and low risk for sudden infant death syndrome. J Pediatr. 1998;132(2):234-9.

Kuhlmann S, Ahlers-Schmidt CR, Lukasiewicz G, Truong TM. Interventions to improve safe sleep among hospitalized infants at eight Children's hospitals. Hosp Pediatr. 2016;6(2):88-94.

Leong T, Billaud M, Agarwal M, Miller T, McFadden T, Johnson J, et al. As easy as ABC: evaluation of safe sleep initiative on safe sleep compliance in a freestanding pediatric hospital. Inj Epidemiol. 2019;6(Suppl 1):26.

Macklin JR, Gittelman MA, Denny SA, Southworth H, Arnold MW. The EASE Quality Improvement Project: Improving Safe Sleep Practices in Ohio Children's Hospitals. Pediatrics. 2016;138(4).

Macklin JR, Gittelman MA, Denny SA, Southworth $H$, Arnold MW. The EASE project revisited: improving safe sleep practices in Ohio birthing and Children's hospitals. Clin Pediatr (Phila). 2019;58(9):1000-7. 
Mason B, Ahlers-Schmidt CR, Schunn C. Improving safe sleep environments for well newborns in the hospital setting. Clin Pediatr (Phila). 2013;52(10):969-75.

McMullen SL, Fioravanti ID, Brown K, Carey MG. Safe sleep for hospitalized infants. MCN Am J Matern Child Nurs. 2016:41(1):43-50.

Moon RY. SIDS and other sleep-related infant deaths: expansion of recommendations for a safe infant sleeping environment. Pediatrics. 2011; 128(5):e1341-67.

Moon RY. SIDS and Other Sleep-Related Infant Deaths: Evidence Base for 2016 Updated recommendations for a safe infant sleeping environment. Pediatrics. 2016;138(5).

National Institute of Child Health and Human Development/National Institutes of Health Safe to Sleep Campaign. Available from: https://safetosleep.nichd.nih. gov/. Cited 201915 September.

Patel AL, Harris K, Thach BT. Inspired CO(2) and O(2) in sleeping infants rebreathing from bedding: relevance for sudden infant death syndrome. J Appl Physiol (1985). 2001;91(6):2537-45.

Patton C, Stiltner D, Wright KB, Kautz DD. Do nurses provide a safe sleep environment for infants in the hospital setting? An integrative review. Adv Neonatal Care. 2015;15(1):8-22.

Scheers NJ, Dayton CM, Kemp JS. Sudden infant death with external airways covered: case-comparison study of 206 deaths in the United States. JAMA Pediatr. 1998:152(6):540-7.

Shadman KA, Wald ER, Smith W, Coller RJ. Improving Safe Sleep Practices for Hospitalized Infants. Pediatrics. 2016;138(3).

Shapiro-Mendoza CK. Interventions to improve infant safe sleep practices. JAMA. 2017;318(4):336-8.

Shapiro-Mendoza CK, Colson ER, Willinger M, Rybin DV, Camperlengo L, Corwin MJ. Trends in infant bedding use: National Infant Sleep Position study, 19932010. Pediatrics. 2015;135(1):10-7.

Wickham H. ggplot2: elegant graphics for data analysis. New York: Springer; 2009.

Zachritz W, Fulmer M, Chaney N. An evidence-based infant safe sleep program to reduce sudden unexplained infant deaths. Am J Nurs. 2016;116(11):48-55.

\section{Publisher's Note}

Springer Nature remains neutral with regard to jurisdictional claims in published maps and institutional affiliations.

Ready to submit your research? Choose BMC and benefit from:

- fast, convenient online submission

- thorough peer review by experienced researchers in your field

- rapid publication on acceptance

- support for research data, including large and complex data types

- gold Open Access which fosters wider collaboration and increased citations

- maximum visibility for your research: over $100 \mathrm{M}$ website views per year

At $\mathrm{BMC}$, research is always in progress.

Learn more biomedcentral.com/submissions 